\title{
The Proton.*
}

\section{By Dr. P. A. M. Dirac, F.R.S.}

$\mathrm{M}$ ATTER is made up of atoms, each consisting of a number of electrons moving round a central nucleus. It is likely that the nuclei are not simple particles, but are themselves made up of electrons, together with hydrogen nuclei, or protons as they are called, bound very strongly together. There would thus be only two kinds of simple particles out of which all matter is built, the electrons, each carrying a charge $-e$, and the protons, each carrying a charge $+e$.

It should be mentioned here that there is a difficulty in this point of view provided by the nitrogen atom. One can infer from the charge and mass of the nitrogen nucleus that it should consist of 14 protons and 7 electrons, but it appears to have properties inconsistent with its being composed of an odd number of simple particles. However, very little is really known about nuclei, and the opinion is generally held by physicists that some way of evading this difficulty will be found and that all nuclei will ultimately be shown to be made up of electrons and protons.

It has always been the dream of philosophers to have all matter built up from one fundamental kind of particle, so that it is not altogether satisfactory to have two in our theory, the electron and the proton. There are, however, reasons for believing that the electron and proton are really not independent, but are just two manifestations of one elementary kind of particle. This connexion between the electron and proton is, in fact, rather forced upon us by general considerations about the symmetry between positive and negative electric charge, which symmetry prevents us from building up a theory of the negatively charged electrons without bringing in also the positively charged protons. Let us examine how this comes about.

The energy $W$ of a particle in free space is determined in terms of its momentum $p$ according to relativity theory by the equation

$$
W^{2} / c^{2}-p^{2}-m^{2} c^{2}=0,
$$

where $m$ is the rest-mass of the particle and $c$ is the velocity of light. This equation can easily be generalised to apply to a charged particle moving in an electromagnetic field and can be used as a Hamiltonian to give the equations of motion of the particle, and thus its possible tracks in space-time.

Now the above equation is quadratic in $W$, allowing of both positive and negative values for $W$. Thus for some of the tracks in space-time the energy $W$ will have positive values and for the others negative values. Of course a particle with negative energy (kinetic energy is referred to throughout) has no physical meaning. Such a particle would have less energy the faster it is moving and one would have to put energy into it to bring it to rest, quite contrary to anything that has ever been observed.

- Based on a paper read before Section A (Mathematical and Physical Science) of the British Association at Bristol on Sept. 8 .

$$
\text { No. 3181, VoL. 126] }
$$

The usual way of getting over this difficulty is to say that the tracks for which $W$ is negative do not correspond to anything real in Nature and are to be simply ignored. This is permissible only provided that for every track $W$ is either always positive or always negative, so that one can tell definitely which tracks are to be ignored. This condition is fulfilled in the classical theory, where $W$ must vary continuously, since $W$ can never be numerically less than $m c^{2}$ and is thus precluded from changing from a positive to a negative value. In the quantum theory, however, discontinuous variations in a dynamical variable such as $W$ are permissible, and detailed calculation shows that $W$ certainly will make transitions from positive to negative values. We can now no longer ignore the states corresponding to a negative energy and it becomes imperative to find some physical meaning for them.

We can deal with these states mathematically, in spite of their being physically nonsense. We find that an electron with negative energy moves in an electromagnetic field in the same way as an ordinary electron with positive energy would move if its charge were reversed in sign, so as to be $+e$ instead of $-e$. This immediately suggests a connexion between negative-energy electrons and protons. One might be tempted at first sight to say that a negative-energy electron is a proton, but this, of course, will not do, since protons certainly do not have negative kinetic energy. We must therefore establish the connexion on a different basis.

For this purpose we must take into consideration another property of electrons, namely, the fact that they satisfy the exclusion principle of Pauli. According to this principle, it is impossible for two electrons ever to be in the same quantum state. Now the quantum theory allows only a finite number of states for an electron in a given volume (if we put a restriction on the energy), so that if only one electron can go in each state, there is room for only a finite number of electrons in the given volume. We thus get the idea of a saturated distribution of electrons.

Let us now make the assumption that almost all the states of negative energy for an electron are occupied, and thus the whole negative-energy domain is almost saturated with electrons. There will be a few unoccupied negative-energy states, which will be like holes in the otherwise saturated distribution. How would one of these holes appear to our observations? In the first place, to make the hole disappear, which we can do by filling it up with a negative-energy electron, we must put into it a negative amount of energy. Thus to the hole itself must be ascribed a positive energy. Again, the motion of the hole in an electromagnetic field will be the same as the motion of the electron that would fill up the hole, and this, as we have seen, is just the motion of an ordinary particle with a charge $+e$. These two facts make it reasonable to assert that the hole is a proton. 
In this way we see the proper rôle to be played by the negative-energy states. There is an almost saturated distribution of negative-energy electrons extending over the whole of space, but owing to its uniformity and regularity it is not directly perceptible to us. Only the small departures from perfect uniformity, brought about through some of the negative-energy states being unoccupied, are perceptible, and these appear to us like particles of positive energy and positive charge and are what we call protons.

This theory of the proton involves certain difficulties, which will now be discussed. The theory postulates the existence everywhere of an infinite number of negative-energy electrons per unit volume, and thus an infinite density of electric charge. According to Maxwell's equations, this would give rise to an infinite electric field. We can easily avoid this difficulty by a re-interpretation of Maxwell's equations. A perfect vacuum is now to be considered as a region in which all the states of negative energy and none of those of positive energy are occupied. The electron distribution in such a region must be assumed to produce no field, and only the departures from this vacuum distribution can produce a field according to Maxwell's equations. Thus, in the equation for the electric field $E$

$$
\operatorname{div} E=-4 \pi \rho,
$$

the electric density $\rho$ must consist of a charge $-e$ for each state of positive energy that is occupied, together with a charge $+e$ for each state of negative energy that is unoccupied. This gives complete agreement with the usual ideas of the production of electric fields by electrons and protons.

A second difficulty is concerned with the possible transitions of an electron from a state of positive energy to one of negative energy, which transitions were the original cause of our having to give a physical meaning to the negative-energy states. These transitions are very much restricted when nearly all the negative-energy states are occupied, since an electron in a positive-energy state can then drop only into one of the unoccupied negativeenergy states. Such a transition process would result in the simultaneous disappearance of an ordinary positive-energy electron and a hole, and would thus be interpreted as an electron and proton annihilating one another, their energy being emitted in the form of electromagnetic radiation.

There appears to be no reason why such processes should not actually occur somewhere in the world. They would be consistent with all the general laws of Nature, in particular with the law of conservation of electric charge. But they would have to occur only very seldom under ordinary conditions, as they have never been observed in the laboratory. The frequency of occurrence of these processes according to theory has been calculated independently by several investigators, with neglect of the interaction between the electron and proton (that is, the Coulomb force between them). The calculations give a result much too large to be true. In fact, the order of magnitude is altogether wrong. The explanation of this discrepancy is not yet known. Possibly the neglect of the interaction is not justifiable, but it is difficult to see how it could cause such a very big error.

Another unsolved difficulty, perhaps connected with the previous one, is that of the masses. The theory, when one neglects interaction, requires the electron and proton to have the same mass, while experiment shows the mass ratio to be about 1840 . Perhaps when one takes interaction into account the theoretical masses will differ, but it is again difficult to see how one could get the large difference required by experiment.

An idea has recently been put forward by Oppenheimer (Phys. Rev., vol. 35, p. 562) which does get over these difficulties, but only at the expense of the unitary theory of the nature of electrons and protons. Oppenheimer supposes that all, and not merely nearly all, of the states of negative energy are occupied, so that a positive-energy electron can never make a transition to a negativeenergy state. There being now no holes which we can call protons, we must assume that protons are really independent particles. The proton will now itself have negative-energy states, which we must again assume to be all occupied. The independence of the electron and proton according to this view allows us to give them any masses we please, and further, there will be no mutual annihilation of electrons and protons.

At present it is too early to decide what the ultimate theory of the proton will be. One would like, if possible, to preserve the connexion between the proton and electron, in spite of the difficulties it leads to, as it accounts in a very satisfactory way. for the fact that the electron and proton have charges equal in magnitude and opposite in sign. Further advances in the theory of quantum electrodynamics will have to be made before one can deal accurately with the interaction and see whether it will settle the difficulties, or whether, perhaps, a new idea can be introduced which will answer this purpose.

\section{Physiological Effects of Work in Compressed Air.}

THE average man always finds it surprising that 1 our bodies can support such atmospheric pressures as $100 \mathrm{lb}$. per sq. in. without the slightest derangement of the delicate structures and processes on which life depends, but that, owing to a secondary effect, the return to normal pressure is accompanied by grave risk. A sojourner in compressed air inevitably soaks up a considerable volume of the nitrogen of the air into simple solu- tion in the tissues and fluids of his body. So long as the pressure is maintained this gas remains hidden and harmless, but any reduction of pressure will drive it out of solution. The critical time in the management of compressed air workers is the period of decompression when they are passing from high air pressures down to the normal. Given time, the blood will carry off the excess gas and discharge it to the atmosphere in the lungs as the

$$
\text { No. 3181, VoL. 126] }
$$

